\title{
Atomic helium scattering and diffraction from solid surfaces
}

\author{
W H WEINBERG \\ Division of Chemistry and Chemical Engineering, California Institute of Technology, \\ Pasadena, California 91109, USA
}

MS received 13 April 1972

\begin{abstract}
It is shown that whether or not diffractive scattering is observed from solid surfaces depends not only on the elastic scattering cross section, ie the normalized Debye-Waller factor, as has been recently suggested by J L Beeby but also on the surface structure or local surface potential of the particular solid.
\end{abstract}

\section{Introduction}

In a recent very interesting letter, Beeby (1971) has examined theoretically the interaction of helium atoms with solid surfaces. The purpose of his investigation was to determine on which surfaces diffractive (elastic) scattering might be expected to be observed and, in particular, why diffractive scattering is the rule on alkali halide crystal surfaces and the exception on metal surfaces. In the present communication, the results and conclusions of Beeby (1971) are examined in somewhat more detail which allow further generalizations to be made regarding on which surfaces atomic diffraction might be observed, as well as the relative importance of the thermal motion of the solid (DebyeWaller factor) and the local interatomic potential which an impinging gas atom experiences.

\section{Theory}

The thermal motion of a solid surface leads to an enhancement of the inelastic scattering cross section at the expense of the elastic cross section. In fact, the elastic cross section is reduced by the normalized Debye-Waller factor, that is the interatomic potential between the impinging gas atom and the surface is smeared out by the thermal motion of the surface atoms. The Debye-Waller factor may be written as

$$
\delta \equiv \exp \left(-\frac{Q_{\perp}^{2}\left\langle u_{\perp}^{2}\right\rangle}{\hbar^{2}}\right)
$$

where $Q_{\perp}$ is the momentum transfer of the scattered atom orthogonal to the surface, $\left\langle u_{-}^{2}\right\rangle$ is the mean square displacement of a surface atom also in a direction orthogonal to the surface plane, and $\hbar$ is the Planck constant divided by $2 \pi$.

Beeby (1971) has analysed the gas-surface interaction using the time dependent Schrödinger equation in the interaction representation and has shown that the time 
variation of the potential is small all the way down to interaction distances of the order of $0.6 \AA$. This means that the time dependence of the attractive part of the potential can be ignored, that is the time dependence is too rapid for the system to respond to it. Physically, this is a result of a weak, slowly varying potential, and a many-body interaction (one influenced by a large number of surface atoms). The important conclusion which may be drawn from this result is that the attractive part of the potential is effectively time independent, and the thermal motion of the surface atoms only smears out the repulsive part of the potential. It is thus the repulsive part of the potential which determines $\delta$. This is in agreement with recent helium scattering experiments from $W(110)$ (Yamamoto and Stickney 1970, Weinberg and Merrill 1972a) and Pt(111) (Stoll et al 1971) in which the specularly reflected intensity increased at more grazing scattering angles, that is as the scattering angle measured from the surface normal increased. It has been argued that this variation in reflected helium intensity with scattering angle is a result of an interaction principally through the repulsive part of the potential (Weinberg and Merrill 1972a).

The above results are also in agreement with a classical perturbation theory of Goodman (1963) which indicated that the gas-surface collision is languid, and while the attractive interaction is effectively conservative in itself, it is important since it leads to the repulsive interaction being more dissipative. The calculations of Beeby (1971) and Goodman (1963) and the experimental results of Stickney and co-workers and Merrill and co-workers indicate that the momentum transfer (to be used in calculating $\delta$, for example) should be evaluated after the gas atom is accelerated by entering the attractive potential well. This result was used by Beeby (1971) to examine diffractive scattering via the DebyeWaller factor and was used earlier by Weinberg and Merrill (1971) in estimating the probability that an impinging gas atom will be trapped in the gas-surface potential well and will then be re-emitted diffusely, that is, with a cosine distribution.

Beeby (1971) has found that $Q_{\perp}^{2}$ to be used in (1) is given by

$$
Q_{\perp,}^{2} \geqslant 8 m D
$$

where $m$ is the mass of the gas atom, and $D$ is the gas-surface potential well depth. The mean square displacement of a surface atom orthogonal to the surface plane for a Debye solid is given by

$$
\left\langle u_{\perp}^{2}\right\rangle=\frac{3 \hbar^{2} T_{\mathrm{S}}}{M k \theta_{\mathrm{D}, \mathrm{S}}^{2}}\left\{\frac{\theta_{\mathrm{D}, \mathrm{S}}}{4 T}+\phi\left(\frac{\theta_{\mathrm{D}, \mathrm{S}}}{T_{\mathrm{S}}}\right)\right\}
$$

where $T_{\mathrm{S}}, M, k$ and $\theta_{\mathrm{D}, \mathrm{s}}$ are the surface temperature, the mass of a surface atom, the Boltzmann constant and the surface Debye temperature, respectively. The function

$$
\frac{\theta_{\mathrm{D}, \mathrm{S}}}{4 T_{\mathrm{S}}}+\phi\left(\frac{\theta_{\mathrm{D}, \mathrm{S}}}{T_{\mathrm{S}}}\right)
$$

is given as a function of $\theta_{\mathrm{D} \text {. } \mathrm{S}} / T_{\mathrm{S}}$ by James (1965) and may be approximated by unity for $\theta_{\mathrm{D}, \mathrm{S}} \lesssim T$. Combining (1)-(3), using the equality in (2), and assuming the bracketed term in (3) is unity, the following expression for $\delta$ is obtained

$$
\delta=\exp \left(\frac{-24 D T_{\mathrm{S}} \mu^{*}}{k \theta_{\mathrm{D}, \mathrm{S}}^{2}}\right)
$$

where $\mu^{*}$ is the mass ratio, $m / M$. Equation 4 may be used to calculate $\delta$ for experimental conditions appropriate to those reliable scattering experiments which have been reported. 
These calculated values of $\delta$ are shown in table 1 together with the experimental result of whether or not diffraction is observed. The values of the surface Debye temperature are either the experimental values of Morabito et al (1969) or are taken as $58 \%$ of the bulk value of the Debye temperature. The latter derives from the average value found by Morabito et al (1969) for the systems on which they report: the surface Debye tempera -

Table 1. Comparison of calculations with experimental helium scattering distributions

\begin{tabular}{|c|c|c|c|c|}
\hline Surface & $\theta_{\mathbf{D}, S}(\mathbf{K})$ & $T_{\mathrm{s}}(\mathrm{K})$ & $\delta$ & Experimental result \\
\hline $\mathrm{Au}(111)$ & $102^{a}$ & 575 & $0 \cdot 018$ & No diffraction ${ }^{e}$ \\
\hline $\operatorname{Ag}(111)$ & $155^{b}$ & 575 & 0.041 & No diffraction $f$ \\
\hline $\operatorname{Ag}(100)$ & $104^{b}$ & 575 & 0.0058 & No diffraction ${ }^{q}$ \\
\hline $\mathrm{Ni}(111)$ & $220^{b}$ & 700 & 0.029 & No diffraction ${ }^{h}$ \\
\hline Pt (111) & $110^{b}$ & $\geqslant 375$ & $\leqslant 0 \cdot 10$ & No diffraction ${ }^{i, j}$ \\
\hline $\operatorname{Pt}(100)$ & $110^{b}$ & $\geqslant 450$ & $\leqslant 0.065$ & No diffraction ${ }^{k}$ \\
\hline$W(110)$ & $180^{a}$ & $\geqslant 375$ & $\leqslant 0.40$ & No diffraction ${ }^{j, l, m}$ \\
\hline$W(112)$ & $180^{a}$ & $\begin{array}{l}1300 \\
-1900\end{array}$ & $\begin{array}{r}0.043 \\
-0.010\end{array}$ & Diffraction $^{n}$ \\
\hline$C(100)$ diamond & $1080^{a}$ & $\geqslant 300$ & $\leqslant 0.77$ & No diffraction ${ }^{c, j}$ \\
\hline $\begin{array}{l}\mathrm{W}(110)-\mathrm{R}(3 \times 5) 35^{\circ} \\
\text { carbide }\end{array}$ & $1230^{c, d}$ & $375-1300$ & $0.78-0.43$ & Diffraction $^{d, j, p}$ \\
\hline $\operatorname{LiF}(100)$ & $508^{a}$ & 300 & $\geqslant 0.24$ & Diffraction ${ }^{q}$ \\
\hline $\mathrm{NaCl}(100)$ & $179^{a}$ & $300-580$ & $\sim 0.0022$ & Diffraction ${ }^{q}$ \\
\hline
\end{tabular}

a Surface Debye temperature taken as $58 \%$ of bulk value. Bulk values taken from Guinier (1963), James (1965) and de Launay (1956)

${ }^{b}$ Morabito et al (1969)

c Weinberg (1971)

${ }^{d}$ Weinberg and Merrill (1972b)

e Smith and Saltsburg (1964)

$f$ Saltsburg and Smith (1966)

g Palmer et al (1970)

${ }^{h}$ Smith et al (1969)

i Stoll et al (1971)

j Weinberg and Merrill (1972c)

k West and Somorjai (1971)

'Yamamoto and Stickney (1970)

${ }^{m}$ Weinberg and Merrill (1972a)

${ }^{n}$ Tendulkar and Stickney (1971)

${ }^{q}$ A series of papers in Z. Phys. commencing with Estermann and Stern (1930)

Following Beeby (1971), $D=300 \mathrm{cal} \mathrm{mol}^{-1}$ for $\mathrm{He}-$ metal interactions, and $D=250 \mathrm{cal} \mathrm{mol}^{-1}$ for $\mathrm{He}-\mathrm{LiF}, \mathrm{He}-\mathrm{NaCl}, \mathrm{He}-\mathrm{C}$ and $\mathrm{He}-\mathrm{WC}$ interactions.

ture is found to lie between $\frac{1}{2}$ and $\frac{2}{3}$ of the bulk value. The experimental determination is made using the Debye-Waller attenuation of low energy electron diffraction peaks. The value of the gas-metal surface potential well depth, $D$, is taken as $300 \mathrm{cal} \mathrm{mol}^{-1}$ by assuming one conduction electron per atom in the formulation of Margenau and Pollard (1941) and Pollard (1941). This is the same value used by Beeby (1971). The use of one conduction electron per surface atom for all the metals listed in tables 1 and 2 is a first approximation since the number of conduction electrons varies from metal to metal in an unknown fashion. The number of bonding electrons, $n$, in the various transition metals based on the Engel-Brewer correlation (Brewer 1968) is shown in table 2 . It is the bonding electrons which result in the high cohesive energy of the transition metals. However, at a surface these normally bonding electrons may occupy surface orbitals and greatly increase the very small number of holes usually associated with the 
$\mathrm{d}$ band in bulk transition metals (eg de Haas-Van Alphen experiments have placed an upper limit of $10^{-4}$ holes in the $\mathrm{d}$ band for platinum (Mueller et al 1970, Andersen 1970)). The available surface orbitals are also a function of crystallographic orientation so that the appropriate number of 'conduction electrons' to use in the formulation of Margenau

Table 2. Calculated Debye-Waller factors for metal surfaces at $T_{\mathrm{S}}=375 \mathrm{~K}$

\begin{tabular}{llll}
\hline Surface & $n$ & $\theta_{\mathrm{D}, \mathrm{S}}(\mathrm{K})$ & $\delta$ \\
\hline $\mathrm{W}$ & 6 & $180^{a}$ & $0 \cdot 404$ \\
$\mathrm{Ir}$ & 5 & $165^{a}$ & $0 \cdot 357$ \\
$\mathrm{Mo}$ & 6 & $220^{a}$ & $0 \cdot 314$ \\
$\mathrm{Cr}$ & 6 & $267^{a}$ & $0 \cdot 234$ \\
$\mathrm{Fe}$ & 6 & $250^{a}$ & $0 \cdot 215$ \\
$\mathrm{Ta}$ & 5 & $131^{a}$ & $0 \cdot 176$ \\
$\mathrm{Co}$ & 5 & $224^{a}$ & $0 \cdot 162$ \\
$\mathrm{Ni}$ & 4 & $220^{b}$ & $0 \cdot 150$ \\
$\mathrm{~V}$ & 5 & $226^{a}$ & $0 \cdot 126$ \\
$\mathrm{Ag}$ & $2 \cdot 4$ & $155^{b}$ & $0 \cdot 124$ \\
$\mathrm{Nb}$ & 5 & $160^{a}$ & $0 \cdot 104$ \\
$\mathrm{Pt}$ & 4 & $110^{b}$ & 0.102 \\
$\mathrm{Cu}$ & $2 \cdot 4$ & $186^{a}$ & 0.085 \\
$\mathrm{Pd}$ & $3 \cdot 4$ & $140^{b}$ & 0.075 \\
$\mathrm{Au}$ & $2 \cdot 4$ & $102^{a}$ & 0.071 \\
$\mathrm{~Pb}$ & & $55^{b}$ & 0.0002 \\
\hline
\end{tabular}

a Surface Debye temperatures taken as $58 \%$ of bulk value. References as table 1. ${ }^{b}$ Morabito et al (1969).

As in table $1, D=300 \mathrm{cal} \mathrm{mol}^{-1}$ for all He-metal interactions (Beeby 1971).

and Pollard (1941) and Pollard (1941) may not even be exactly constant for a given metal (Weinberg and Merrill 1972d). Evidence for this assertion comes from the measured changes in surface potential for xenon physically adsorbed on various crystallographic orientations of $\mathrm{Ni}$ (Baker and Johnson 1972) and the experimental binding energies of argon, krypton and xenon on various crystal planes of W (Engel and Gomer 1970). Presumably, the appropriate number of conduction electrons to be used in calculating $D$ is a structure factor multiplied by the number of bonding electrons $n$ given in table 2 . The structure factor depends on the number of unsaturated surface orbitals and is a function of crystallographic orientation for a given transition metal. Depending on the number of near neighbours which are vacated in forming the surface, the value of the structure factor would be expected to lie between about $\frac{1}{6}$ and $\frac{1}{2}$. However, it seems clear that the theory is not precise enough to warrant any assumption other than that of one conduction electron per surface atom originally used by Beeby (1971).

\section{Results}

In table 1, there is a natural grouping of the solids into three separate categories: $(a)$ 
metals, which include $\mathrm{Au}, \mathrm{Ag}, \mathrm{Ni}, \mathrm{Pt}$ and $\mathrm{W} ;(b)$ two different forms of carbon, both diamond and a metal with an ordered carbon overlayer; and (c) alkali halides, which include $\mathrm{LiF}$ and $\mathrm{NaCl}$. The results presented in table 1 are useful in assessing the effect of $\delta$ on whether or not atomic helium diffraction is observed from a particular solid surface. It is seen that, in general, metals do not show diffraction effects; and also, in general, $\delta$ for metals is rather small in agreement with the earlier arguments of Beeby (1971). However, Weinberg and Merrill (1972a, c) have observed no diffraction from a W(110) surface at a surface temperature which corresponds to $\delta=0 \cdot 40$, whereas Tendulkar and Stickney (1971) have observed diffraction from a W(112) surface at a surface temperature corresponding to a $\delta$ as small as 0.01. Also, Tendulkar and Stickney (1971) only observed first order diffraction peaks from their (112) surface when the azimuthal angle of the incident helium beam relative to the surface lattice corresponds to the [110] direction. No first order diffraction peaks were observed when the azimuthal angle is in the [111] direction of the surface lattice. Thus, diffraction was observed in a direction in which the surface structure is much more pronounced (across rather than along the rows of $\mathrm{W}$ atoms on the (112) surface). These results point out the evident importance of the surface structure, that is the local surface potential, in addition to the importance of $\delta$.

The importance of surface geometry is also manifested in the helium scattering from a diamond (100) and a tungsten carbide surface on which the highly concentrated surface carbon is ordered into a $(3 \times 5)$ array rotated $35^{\circ}$ with respect to the underlying $W(110)$ substrate (Weinberg 1971, Weinberg and Merrill 1970,1972b, c). Although $\delta$ has approximately the same value and is quite large in both cases, the scattering from the two surfaces is radically different: both first and second order atomic diffraction peaks are observed from the tungsten carbide (Weinberg and Merrill 1970, 1972b, c); whereas the scattering from the diamond (100) surface is quite diffuse, very nearly cosine (Weinberg 1971, Weinberg and Merrill 1972c). The latter result is undoubtedly due to the very open geometrically rough nature of the diamond (100) surface and points up the importance of considering surface structure or local surface potentials in addition to $\delta$ when deciding in which cases diffraction might be observable. Finally, diffraction has been observed from the alkali halides, $\mathrm{LiF}$ and $\mathrm{NaCl}$ which are listed in table 1 (Estermann and Stern 1930). That it has been observed for $\mathrm{NaCl}$ with a $\delta$ of $<0.003$ points to the fact that the local surface potential is of great importance in the scattering of helium from the alkali halides. That the intensity of the diffraction peaks are of the order expected by the $\delta$ values $(\mathrm{LiF}>\mathrm{NaCl})$ points to the importance of $\delta$. There is clearly an effect from both sources: the surface structure and local potential, and the Dcbye-Waller factor.

With the observation of diffractive scattering from W(112) by Tendulkar and Stickney (1971), the attempt to observe diffravive scattering of helium from other metals will undoubtedly occupy the attention of a number of other experimentalists including the present author. In table 2 values of $\delta$ are calculated for a number of different metals, all at the same assumed surface temperature of $375 \mathrm{~K}$. It is seen that although W is the metal most likely to show diffractive scattering, the elastic cross section remains above $20 \%$ for $\mathrm{Ir}, \mathrm{Mo}, \mathrm{Cr}$ and $\mathrm{Fe}$ as well as W. Table 2 should be of value to experimentalists as they decide which metals might be used in further attempts to observe atomic diffraction.

\section{Conclusions}

Based on experimental data presently available, it has been shown that both the surface structure (local surface potential) as well as the elastic cross section (Debye-Waller 
factor, $\delta$ ) are important in determining the helium scattering distributions from solid surfaces. Finally, two sets of experiments are suggested which should help to evaluate the relative importance of these two effects for the interesting case of metal surfaces. First, appropriate crystal orientations must be chosen. The FCC (110) and BCC (112) crystal planes are very convenient to use since they are quite similar and diffraction has been previously observed on a BCC (112) surface. The intensity of diffracted helium beams should decrease in the order (at constant $T_{\mathrm{s}}$ ) W (112) $>\operatorname{Ir}(110)>$ Mo $(112)>$ $\mathrm{Cr}(112)>\mathrm{Fe}(112)>\mathrm{Ta}(112)$ etc for the metals listed in table 2. This experiment evaluates the effect of $\delta$ for similar surface structures and local potentials. Secondly, a given metal such as W is chosen, and helium is scattered from different crystal planes at constant $T_{\mathrm{S}}$ in order to determine the effect of the surface structure or local potential at a constant $\delta$. As mentioned above, this experiment has been partially completed with no diffraction observed from W (110) at $T_{\mathrm{S}}=375 \mathrm{~K}(\delta=0.40)$ (Weinberg and Merrill $1972 \mathrm{a})$, but with diffraction observed from W (112) up to $1900 \mathrm{~K}(\delta=0.010)$ (Tendulkar and Stickney 1971). This result together with the anisotropic scattering results on W (112) already points up the importance of the local surface structure. The fact that no diffraction is observed from FCC (111) or (100) crystal planes and BCC (110) plane is undoubtedly due to the fact they are the smoothest possible metal surfaces. Other crystal orientations of intermediate roughness between those mentioned above and the BCC (112) should be chosen to determine the surface structure necessary for diffraction to be observed.

Finally, it should be emphasized that the gas-surface potential well depth for the interaction of helium with the various metal surfaces was assumed equal to the constant value of $300 \mathrm{cal} \mathrm{mol}^{-1}$, that is one conduction electron per metal surface atom was used in connection with the theory of Pollard (1941). When experimentally measured initial heats of physical adsorption become available (ideally as a function of crystallographic orientation) those values for $D$ may be used in equation 4 in order to calculate $\delta$. It is possible that the ordering of the metals in table 2 will then have to be slightly revised. It is even possible that inferences may be made concerning the relative gas-surface potential well depths from observed variations in intensity of atomic diffraction beams.

\title{
Acknowledgment
}

Financial support from the Division of Chemistry and Chemical Engineering of the California Institute of Technology is gratefully acknowledged.

\author{
References \\ Andersen O K 1970 Phys. Rev. B $2883-906$ \\ Baker B G and Johnson B B $1972 \mathrm{~J}$. Vac. Sci. Technol. 9 930-3 \\ Beeby J L 1971 J. Phys. C: Solid St. Phys. 4 L359-62 \\ Brewer L 1968 Science 161 115-22 \\ Engel T and Gomer R $1970 \mathrm{~J}$. chem.Phys. 52 5572-80 \\ Estermann I and Stern O 1930 Z. Phys. 61 95-103 \\ Goodman F O 1963 J. Phys. Chem. Solids 24 1451-66 \\ Guinier A 1963 X ray Diffraction (San Francisco: Freeman) \\ James R W 1965 Optical Principles of the Diffraction of $X$ rays (London: Bell) \\ de Launay J 1956 Solid State Phys. 2219-303 (New York: Academic Press) \\ Margenau H and Pollard W G 1941 Phys. Rev. 60 128-34 \\ Morabito J M Jr, Steiger R F and Somorjai G A 1969 Phys. Rev. 179 638-43
}


Mueller F M, Freeman A J, Dimmock J O and Furdyna A M 1970 Phys. Rev. B 1 4617-35

Palmer R L, O'Keefe D R, Saltsburg H and Smith J N Jr $1970 \mathrm{~J} \quad$ Sci. Technol. 791-5

Pollard W G 1941 Phys. Rev. 60 578-85

Saltsburg H and Smith J N Jr 1966 J. chem. Phys. 45 2175-83

Smith J N Jr and Saltsburg H 1964 J, chem. Phys. 40 3585-91

Smith J N Jr, Saltsburg H and Palmer R L 1969 Rarefied Gas Dynamics, 6th Symp. vol II eds L Trilling and H Y Wachman (New York: Academic Press) pp 1141-57

Stoll A G, Smith D I and Merrill R P 1971 J. chem. Phys. $54163-9$

Tendulkar D V and Stickney R E 1971 Surface Sci. 27 516-22

Weinberg W H 1971 PhD Thesis, Chemical Engineering Dept,University of California, Berkeley

Weinberg W H and Merrill R P 1970 Phys. Rev. Lett. 25 1198-201

- $1971 \mathrm{~J}$. Vac. Sci. Technol. $8718-24$

_- 1972a J. chem. Phys. 56 2281-92

- 1972 b J. chem. Phys. $562893-902$

—_ 1972c, Proceedings 2nd Int. Symp. Adsorption-Desorption Phenomena (New York: Academic Press) to be published

1972d Surface Sci. (submitted)

West L A and Somorjai G A $1971 \mathrm{~J}$. chem. Phys. 54 2864-73

Yamamoto S and Stickney R E 1970 J. chem. Phys. 53 1594-604 